\title{
Pilonidal Sinus Carcinoma dying from Squamous Cell Carcinoma within 14 months after Diagnosis.
}

\author{
Marius Dettmer ${ }^{1,2}$, Marcel Bonni MD ${ }^{2,3}$, Kostantinos Degiannis MD ${ }^{2,4}$, Matthias Maak MD 5 , \\ Dietrich Doll MD, $\mathrm{PhD}^{1,2,6, *}$, Igors Iesalnieks $\mathrm{MD}^{7}$
}

Received: 08 December 2021 / Accepted: 06 January 2022 / Published online: 20 January 2022

This article is published with open access at https://journal.astes.org.al

(C) The author(s) 2022. \& Copyright (C) 2022, the Albanian Society for Trauma and Emergency Surgery

(c) The Albanian Journal of Trauma and Emergency Surgery is an Open Access Journal. All articles are distributed under the terms of the Creative Commons Attribution Non-Commercial License: http://creativecommons.org/licenses/by-nc/4.0/) which permits unrestricted non-commercial use, distribution, and reproduction in any medium provided the original work is properly cited.

\begin{abstract}
Introduction: The incidence of Pilonidal Sinus Disease (PSD) is increasing worldwide, especially in the developing and developed countries. As long-standing chronic infection may trigger neoplastic transformation, more carcinoma arising from PSD are to be expected with an incidence of $0,1 \%$.

Methods: Case report describing a Squamous cell carcinoma (SCC) arising from PSD

Results: A 60 -year-old male presented with a $6 \mathrm{~cm}$ x $10 \mathrm{~cm}$ ulcerating wound in the sacrococcygeal area, which was painful for 3 months. A biopsy initially revealed a highly differentiated squamous cell carcinoma (cT3cN1acM0G1). Soft tissue MRI showed possible infiltration of the coccyx, and a staging $\mathrm{CT}$ showed suspicious enlarged lymph nodes within both inguinal regions. The patient underwent a full 20x1,8 Gy chemoradiation with partial response of the primary. Surgical Resection was recommended. The patient did not show up for follow-up after chemoradiation and refused further treatment. He demised after 14 months due to pulmonary metastases.

Conclusion: Even a curative intended chemoradiation of this highly differentiated tumour led to a dismal outcome of disease after 14 months. Cases of PSD carcinomas often present as atypical cutaneous SCC.

Keywords: Pilonidal Sinus, Pilonidal Carcinoma, Histology, Radiotherapy
\end{abstract}

Original article, no submission or publication in advance or in parallel

\section{* Corresponding author:}

Prof. Dr. med. Dr. phil. Dietrich Doll,

$\bowtie$ Dietrich.Doll@kk-om.de

1 Department of Procto-Surgery, St. Marienhospital Vechta, Academic Teaching Hospital of the MHH Hannover, Vechta, GERMANY

2 Vechtaer Research Institute VIFF, Vechta, GERMANY

3 Department of Internal Medicine, Federal Armed Forces Hospital, Westerstede, GERMANY

4 Department of Trauma, Orthopedic and Reconstructive Surgery, St. Marienhospital Vechta, Academic Teaching Hospital of the MHH Hannover, Vechta, GERMANY

5 Department of Surgery, Friedrich-Alexander University (FAU) Erlangen-Nuremberg and Universitätsklinikum Erlangen, Erlangen, GERMANY.

6 Department of Surgery, Medical School, University of the Witwatersrand, Johannesburg, Republic of SOUTH AFRICA

7 Department of Surgery, Evangelisches Krankenhaus Kalk, Cologne, GERMANY

\section{Introduction}

Pilonidal Sinus Disease (PSD) has an acute or acute-onchronic presentation and affects mostly young males [1]. The etiology is linked to entrance of sharp hair fragments, originating from the occiput[2,3], into the cutis of the sacral region[4]. It is a common, histologically benign condition with a tendency of rising incidence [5]. Development of carcinoma on the ground of PSD has only been described anecdotally with a frequency of one in a thousand patients $(0,1 \%)[6]$.

The case of a patient, who developed squamous cell carcinoma (SCC) on the ground of chronic PSD, is presented and literature of this pathology is reviewed.

This case report looks at a 60 -year-old male patient with a well differentiated PSDCA, who demised 14 months after initial diagnosis. 


\section{Case report}

A 60 year old male patient was admitted in March 2018 with a large, ulcerating tumor in the sacrococcygeal area. He had been suffering from untreated PSD for 25 years, which presented with minimal discharge during its course. Prior to admission, the patient had reported an increased pain in the affected area with deterioration in general condition and weight loss. On clinical inspection, the growth was 6 by 10 $\mathrm{cm}$, exulcerated, partially abscessing with raised margins, and tenderness (Figure 1). An MRI was performed and showed a large infiltration of the coccyx with the growth being $12 \times 5.5 \times 6 \mathrm{~cm}$ (Figure 2). Histology showed a highly differentiated squamous cell carcinoma. An initial staging CT showed no metastases but an increase in size of right inguinal $(2.7 \mathrm{~cm})$ and left inguinal $(2 \mathrm{~cm})$ lymph nodes (Figure 3). Initial clinical TNM classification was therefore cT3cN1acM0G1, analogue to the anal rim carcinoma. Per recommendation by a multidisciplinary tumor board, the patient was started on a 3-month course of neoadjuvant chemoradiation. The radiotherapy scheme was as follows: 59.4 Gy over the tumor, 50.4 Gy over the perirectal and iliac lymphatics, inguinal right and inguinal left each. Enlarged lymph nodes inguinal right and left received 9 Gy boost each. Chemotherapy used was Mitomycin $10 \mathrm{mg} / \mathrm{m}^{2} / \mathrm{d} 1$ and 5-FU 750mg/m² $11-5$.

The patient received chemoradiation until May 2018 with partial response of the tumor. A surgical resection was scheduled. Unfortunately, the patient did not show up for follow-up after chemoradiation and refused surgery. His condition deteriorated fast through the summer and he demised in February 2019 with pulmonary metastasis.

\section{Discussion}

We report the $128^{\text {th }}$ published case of carcinoma arising from PSD which had a fast progression leading to death with pulmonary metastases. Incidence of PSDCA is considered to be about $0,1 \%$ [6]. Incidence of PSD is increasing over the last years [5] this might lead to rising numbers of carcinomas arising PSD. [7] But this cannot be seen as proven by only 128 cases described in literature.

PSDCA typically affects patients with an average age of 52 years [8] with a long history of Pilonidal Sinus Disease (mean duration 22 years) [7]. Histologically confirmed SCC has been found in $75 \%$ of all patients[9] with sweat gland adenocarcinoma, basal cell carcinoma and verrucous carcinoma being less frequent $[6,10]$.

Thus PSDCA may arise in a scenario of long standing infection, causing change in the tissue architecture, combined with alteration in its immune response. This physical history of malignancy related to longstanding PSD infection is similar to that of other cases of carcinomas, presenting in an area of previously traumatized, chronically inflamed or scarred skin, commonly present in the context of chronic wounds including burn injuries, venous ulcers, ulcers from osteomyelitis and post radiotherapy scars $[8,11]$
In suspicious PSD, a biopsy should be initially taken to rule out malignancy. If biopsy verifies malignancy, more diagnostic is needed. MRI to check for local infiltration and enlarged or suspicious lymph nodes followed by rectoscopy if an invasion is suspected. CT scan should be done to check for distant metastases.

In the present case, the CT showed potential lymph node metastasis (LNM) which is rare in cutaneous squamous cell carcinoma (cSCC)[12]. The prognosis in SCC with LNM is not well studied but described in 14\% of PSDCA and is highly associated in Marjolins Ulcer [8, 13]. The prognosis of SCC in PSD and anal rim carcinoma is significantly correlating with grading of the tumor cells [14].

Many PSDCA lead to death while showing a moderate to well differentiation (over 55\%). Furthermore, SCC of the skin has an overall respective survival rate of $92 \%$ in males and $94.9 \%$ in females at 5 years [15], which is not seen in PSDCA. Hence a further important factor seems to emerge.

The answer to this enigma can be found within the origin of the pilonidal sinus genesis. The typical and common anatomical feature of PSD are one or multiple tracts from the surface ranging into the depth, reaching a pilonidal nest in most cases. The tracts tend to point upwards to the left side and sacral wards into the subcutaneous tissues. Although they nearly never reach the sacrum, the proximity of tracts and presacral fat can be measured in a few millimeters.

There are four factors that promote the malignancy of the PSDCA:

1. First, the tracts give an easy path for tumor cells to spread in deeper tissue.

2. The gluteal and presacral area is characterized by the change between movement and compression. Repetitive tumor compression might lead to tumor cell ablation from the primary and dissemination along the tracts.

3. The presence of decade long lasting infection leads to locally reduced cellular defense mechanisms.

4. Perfusion might be reduced by sitting, thus, tumor cells might move into dormant status of T0. Furthermore, chronic infection around the tracts leads to reduced perfusion, hence reduced access of immunological response to the tumor cells present in the tract.

PSDCA is normally treated like SCC of the skin or anal rim carcinoma. Comparing the described PSDCA and also considering our case, where even highly differentiated carcinoma leads to dismal outcome, it is striking that the used classification systems of anal rim carcinomas or SCC do not seem to be best fitting yet. The special anatomic features of PSDCA outlined above can explain the presentation of PSDCA as atypical cSCC in regard of their metastasis rates and mortality. A comparison of data in carcinomas arising from fistulas like PSDCA may help to investigate whether PSDCA is a late detected SCC of the skin or should be reconsidered as its own entity. Furthermore, our Case showed that chemoradiation as neoadjuvant therapy regime can be helpful to reduce tumor volume getting better surgical conditions as described in previous Case reports. However, no case has been described in the literature where a patient has received chemoradiation as a definitive therapy of PSDCA. 


\section{Conclusions}

Cases of PSD carcinomas often present more malignant as compared to typical $\mathrm{cSCC}$ in terms of their metastasis rates and mortality. The prognostic parameters of PSDCA need to be re-evaluated and differentiated in contrast to other tumours of that anatomical region.

Ethics: Informed consent for publication was obtained from the family of the deceased patient.

Conflicts of interest: All authors declare that they have no conflicts of interest.

Funding: No funding or grants from any other funding agencies in the public, commercial, or not-for profit sectors were received.

\section{References}

1. Luedi, M.M., et al., Global Gender Differences in Pilonidal Sinus Disease: A Random-Effects Meta-Analysis. World J Surg, 2020. 44(11): p. 3702-3709.

2. Bosche, F., et al., The Hair in the Sinus: Sharp-Ended Rootless Head Hair Fragments can be Found in Large Amounts in Pilonidal Sinus Nests. World J Surg, 2018. 42(2): p. 567-573.

3. Doll, D., et al., The presence of occipital hair in the pilonidal sinus cavity-a triple approach to proof. Int J Colorectal Dis, 2018. 33(5): p. 567-576.

4. Downing, J.G., Barbers' pilonidal sinus. J Am Med Assoc, 1952. 148(17): p. 1501.

5. Dettmer, M., et al., Gender differences in axial hair strength may explain gender related incidence variation in Pilonidal Sinus patients. PSJ, 2021. 7(1): p. 11-20.

6. White, T.J., et al., Don't sit on chronic inflammation. ANZ J Surg, 2012. 82(3): p. 181-182.

7. Yuksel, M.E. and F. Tamer, All pilonidal sinus surgery specimens should be histopathologically evaluated in order to rule out malignancy. J Visc Surg, 2019. 156(5): p. 469-470.

8. Abboud, B. and H. Ingea, Recurrent squamous-cell carcinoma arising in sacrococcygeal pilonidal sinus tract: report of a case and review of the literature. DCR, 1999. 42(4): p. 525528.

9. Kulaylat, M.N., M. Gong, and R.J. Doerr, Multimodality treatment of squamous cell carcinoma complicating pilonidal disease. Am Surg, 1996. 62(11): p. 922-929.

10. Mentes, O., M. Akbulut, and M. Bagci, Verrucous carcinoma (Buschke-Lowenstein) arising in a sacrococcygeal pilonidal sinus tract: report of a case. Langenbecks Arch Surg, 2008. 393(1): p. 111-114.

11. Al-Zacko, S.M., Malignancy in chronic burn scar: a 20 year experience in Mosul-Iraq. Burns, 2013. 39(7): p. 1488-91.

12. Silberstein, E., et al., Lymph Node Metastasis in Cutaneous Head and Neck Squamous Cell Carcinoma. Dermatol Surg, 2015. 41(10): p. 1126-9.

13. Shen, R., et al., Clinical characteristics and therapeutic analysis of 51 patients with Marjolin's ulcers. Exp Ther Med, 2015. 10(4): p. 1364-1374.
14. Borges, V.F., et al., Clinicopathologic characterization of squamous-cell carcinoma arising from pilonidal disease in association with condylomata acuminatum in HIV-infected patients: report of two cases. DCR, 2001. 44(12): p. 18731877.

15. Hollestein, L.M., E. de Vries, and T. Nijsten, Trends of cutaneous squamous cell carcinoma in the Netherlands: increased incidence rates, but stable relative survival and mortality 1989-2008. Eur J Cancer, 2012. 48(13): p. 2046-53.

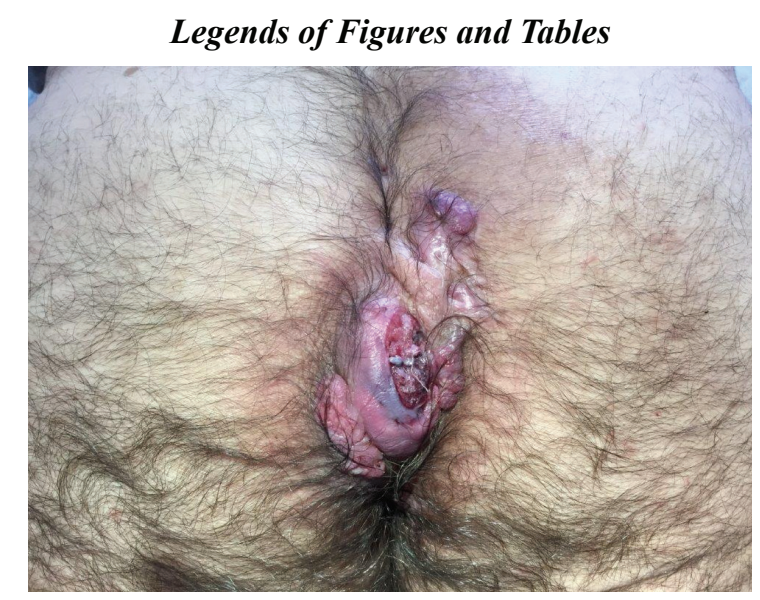

Figure 1: Initial clinical presentation of the ulcerating PSD-CA 6x10cm.

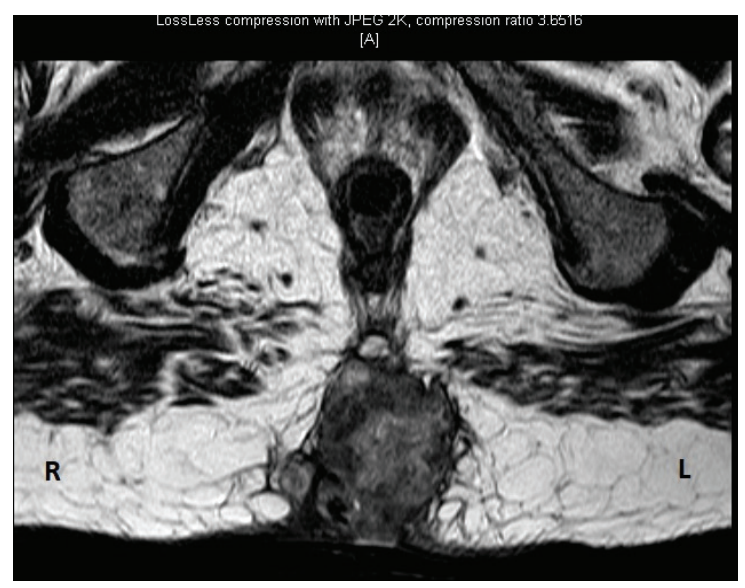

Figure 2: MRI of the pelvis, which shows a large infiltration of the coccyx with the growth being $12 \times 5.5 \times 6 \mathrm{~cm}$.

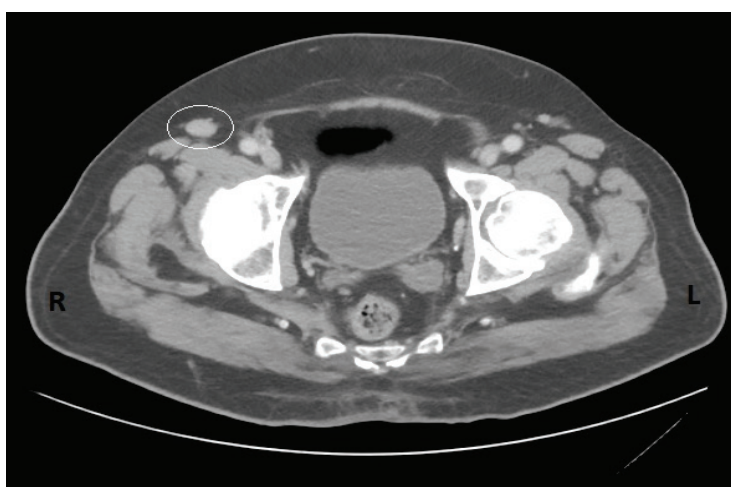

Figure 3: CT-scan which shows enlarged inguinal lymph nodes 